\title{
LOS JÓVENES EN LA ESTRUCTURA DEMOGRÁFICA DE LA REGIÓN METROPOLITANA DE MADRID
}

\author{
POR
}

\author{
JULIO VINUESA ANGULO
}

\section{Introducción}

Uno de los aspectos más relevantes de la dinámica demográfica, especialmente en los ámbitos urbanos y metropolitanos, son las oscilaciones del volumen de población de los distintos intervalos de edad. Sin embargo, no es fácil encontrar análisis referidos a esta cuestión. Sólo la dinámica de los grupos de edad avanzada ha provocado en los últimos años un creciente interés ante el fuerte proceso de envejecimiento demográfico. En este caso se trata de centrar la atención en unos grupos de población que, por el contrario, no han suscitado demasiado interés demográfico. No es el grupo de niños, directamente afectado por la caída de la fecundidad, ni el de los mayores, beneficiado por el aumento de la esperanza de vida. Tampoco se trata de un grupo que haya protagonizado flujos migratorios especialmente intensos. Por el contrario, es un grupo intermedio, no claramente definido y cuya existencia no ha tenido eco ni en los indicadores demográficos más frecuentes en la tradición del análisis demográfico ni tampoco en estudios regionales de población.

$\mathrm{Al}$ margen pues de los estudios al uso, en este trabajo se trata de analizar desde una perspectiva demográfica el segmento juvenil de la po-

J. Vinuesa Angulo. Departamento de Geografía. Universidad Autónoma de Madrid.

Estudios Geográficos

Tomo LXI, 2000, n. ${ }^{\circ} 241$, octubre-diciembre 
blación de la región metropolitana madrileña. Este simple enunciado del propósito general pone de manifiesto los elementos básicos del análisis, pero debe ser completado con algunas consideraciones metodológicas previas.

Se hace referencia a una población específica que hay que definir y que exige una acotación precisa de sus límites de edad. Pero, además, se trata de una población distribuida en un espacio metropolitano, lo cual hace especialmente relevante observar las interdependencias geodemográficas en los ámbitos territoriales de referencia más oportunos en cada caso, tanto para el análisis como para la aplicabilidad de los resultados.

El manejo del concepto de juventud obliga a recurrir a estudios sociológicos que hablan de un periodo de transición, o estadio intermedio entre la infancia y la edad adulta, inherente al proceso de reproducción de toda sociedad. Es una etapa cada vez más amplia que separa el momento en que se alcanza la capacidad fisiológica para realizar las funciones físicas propias de los adultos (procrear, trabajar, defenderse) del momento en que se adquiere la capacidad psicosocial para desempeñarlas (Zárraga, J. L., 1984).

Los primeros estudios sobre juventud establecieron como límites convencionales, internacionalmente aceptados, los 15 y los 24 años, que, como puede apreciarse, se acomodan al típico agrupamiento quinquenal del análisis demográfico. El umbral inferior corresponde, sin coincidir exactamente, con el inicio de la enseñanza secundaria y con la edad mínima legal de incorporación al mercado laboral. El límite superior pretende aproximarse a las fechas de emancipación y por ello, a lo largo del tiempo, se ha ido haciendo más conveniente elevarlo, ampliando el marco de análisis al menos hasta los 29 años. Efectivamente el alargamiento de los estudios, la precarización del empleo, las altas tasas de paro juvenil y el encarecimiento del mercado de la vivienda vienen provocando que la edad del matrimonio vaya experimentando progresivos retrasos, tal como muestran los datos del Cuadro I.

El matrimonio es en nuestra sociedad el verdadero acto de emancipación de los jóvenes (Flaquer, L., 1997) y, por tanto, el momento más adecuado para fijar la edad de salida del periodo juvenil.

La utilización de estos límites debe interpretarse como un intento de aproximación a una realidad sociodemográfica difícil de escudriñar. Con el agrupamiento quinquenal de edades, se delimita entre los 15 y los 29 
CuAdro I

EDAD MEDIA AL MATRIMONIO PARA SOLTEROS

EN LA COMUNIDAD DE MADRID

\begin{tabular}{lcc}
\hline & Hombres & Mujeres \\
\hline 1976 & 26,54 & 24,47 \\
1986 & 27,35 & 25,62 \\
1996 & 29,97 & 28,54 \\
\hline
\end{tabular}

Fuente: Demografía y Salud, n. ${ }^{\circ}$ 44, diciembre de 1998. Comunidad de Madrid.

años un grupo de «jóvenes» que, estando formado en su inmensa mayoría por los individuos que han superado la infancia y aún no han entrado plenamente en la edad adulta, sólo puede considerarse como razonablemente preciso en sus extremos.

Es también oportuno señalar que la amplitud de quince años que se establece no encierra un grupo homogéneo, sino que, por el contrario, incluye individuos con marcadas diferencias en cuanto a sus procesos de formación y de emancipación, que se traducen en distintas necesidades y formas de vida. La consideración individualizada de los tres grupos quinquenales de edad (15-19, 20-24 y 25-29) y, en ocasiones puntuales, el manejo de las edades simples, serán de gran utilidad en el conocimiento de la dinámica y de la estructura de la población de jóvenes.

Establecido el grupo de jóvenes, es necesario señalar cuál debe ser el contenido y cuál la finalidad de su análisis demográfico. El grupo de jóvenes ha sido tradicionalmente considerado en tanto que "promesa». Es la población que en un futuro más o menos próximo habrá de asumir el protagonismo social que corresponde a los individuos de edad adulta. Desde un punto de vista demográfico interesa medir su capacidad de sustitución de otros grupos. Se trata de medir su volumen en términos relativos para poder valorar los procesos de reemplazo y de transferencias entre generaciones. Básicamente interesa centrar la atención en sus posibles efectos sobre la fecundidad y sobre el mercado laboral.

A partir del decenio de los 80, en buena parte por el incremento experimentado entonces por este segmento de edad, adquiere relevan- 
cia la consideración del grupo en relación con distintos problemas, tales como la masificación en las aulas, el paro juvenil, la dificultad de acceso a la primera vivienda, y otros varios como la delincuencia o la drogodependencia. Ante tales circunstancias, se trata de estudiar el grupo en sí mismo, como protagonista de situaciones conflictivas o problemáticas. Es preciso conocer su volumen, su dinámica, su estructura y su comportamiento sociodemográfico como posibles causas de desajustes y tensiones en las estructuras urbanas, generalmente por las oscilaciones de sus demandas y necesidades.

Además de describir demográficamente el propio grupo y su relación con el conjunto de la población, es necesario también determinar los factores causales de su dinámica para concluir señalando algunas de los principales efectos socioeconómicos.

El fenómeno de metropolitanización, que marca intensamente la estructura territorial de la Comunidad de Madrid, representa un papel fundamental en la dinámica demográfica de sus distintos ámbitos. La expansión espacial del proceso urbano de Madrid en coronas concéntricas ha ido produciendo, tras la primera fase de concentración demográfica, una sucesión de desplazamientos de población hacia periferias cada vez más alejadas, que, además de la consiguiente redistribución espacial de la población, ocasionan, en los niveles locales, intensos desequilibrios en la composición por edades y fuertes oscilaciones en las pautas del comportamiento demográfico (Vinuesa, J., 1999). Así, la determinación de las unidades espaciales de análisis tiene, como no podía ser de otra manera, una enorme importancia en este análisis.

La disponibilidad de datos desagregados permite una mayor aproximación a la diversidad de situaciones y comportamientos existentes en los ámbitos municipales. Aún cuando algunos de los efectos derivados de la dinámica demográfica de estos grupos de edad pueden materializarse en necesidades y políticas al nivel regional, es en los municipios donde realmente se localizan y han de gestionarse los problemas de escolarización, paro o vivienda, por citar algunos de los más específicamente juveniles.

Los ámbitos temporales de referencia utilizados son diversos según la disponibilidad de datos y tratan de adaptarse a las exigencias del análisis. Para la valoración de la dinámica de la población, además de los datos de los Censos y Padrones correspondientes, se han considerado los resultados de las últimas proyecciones de población, con horizonte 
en el 2011 (Comunidad de Madrid,1998). Aún cuando los resultados de estas proyecciones para lo ámbitos locales puedan ser discutibles, especialmente en relación con las hipótesis migratorias manejadas, que traducen en tasas constantes los datos de la Estadística de Variaciones Residenciales del periodo 1992-95, se considera que tienen una gran utilidad para la posible aplicabilidad de los resultados de este análisis en estrategias planificadoras.

\section{Una población en franca regresión}

Parece oportuno comenzar con algunas valoraciones generales, aunque posteriormente, al concretar grupos y ámbitos, habrán de ser matizadas. Para el conjunto de la Comunidad de Madrid, el grupo de jóvenes presenta una tendencia claramente regresiva. El Censo de 1991 dio una cifra de 1.279.028 individuos entre 15 y 29 años, marcando su máximo histórico (Cuadro II). Desde 1970 hasta 1991 este grupo venía

CUADRO II

EVOLUCIÓN DEL GRUPO DE JÓVENES EN LA COMUNIDAD DE MADRID

\begin{tabular}{lrc}
\hline & Total & Tasa anual \% \\
\hline 1970 & 868.890 & \\
1975 & 1.008 .779 & 3,03 \\
1981 & 1.102 .353 & 1,79 \\
1986 & 1.178 .031 & 1,34 \\
1991 & 1.279 .028 & 1,66 \\
1996 & 1.278 .743 & 0,00 \\
2001 & 1.183 .183 & $-1,54$ \\
2006 & 1.011 .364 & $-3,09$ \\
2011 & 875.711 & $-2,84$ \\
\hline
\end{tabular}

Fuente: Padrón municipal de 1975. Estadística de Población de la Comunidad de Madrid, 1996. Tomo I: Características Demográficas Básicas. (A través de Anteojo.) Proyecciones de población y de hogares de la Comunidad de Madrid, 1996-2011. Tomo I: Proyecciones básicas por sexo y edad. 
creciendo con unos ritmos superiores a los del conjunto de la población. Se trata de una evolución claramente marcada por los flujos inmigratorios habidos durante este periodo y, sobre todo, en los quinquenios anteriores. Pero a partir de 1996 se dejará notar el efecto del descenso de la fecundidad que se inicia veinte años antes, produciéndose unos fuertes ritmos negativos que divergen claramente de la estacionaridad de la población total (Fig. 1). Obsérvese que entre 1970 y 1991 el grupo de jóvenes aumentó en algo más de 400.000 individuos (lo que representa un crecimiento del 46\%). Tras un breve periodo de estancamiento, a partir de 1996 se inicia la tendencia regresiva que, con ritmos aún más fuertes que en el periodo anterior, reducirá el grupo en otros 400.000 individuos.

Estas fuertes oscilaciones, que como veremos se intensifican en los ámbitos de menor tamaño, tienen una incidencia muy negativa sobre diversos aspectos de la organización social y económica. Por ejemplo, los fuertes crecimientos seguidos de intensas reducciones en la demanda de puestos escolares hacen difícil el correcto funcionamiento del sistema educativo en sus diferentes niveles. Aún cuando más adelante se concretará y se matizará espacialmente esta afirmación, puede decirse

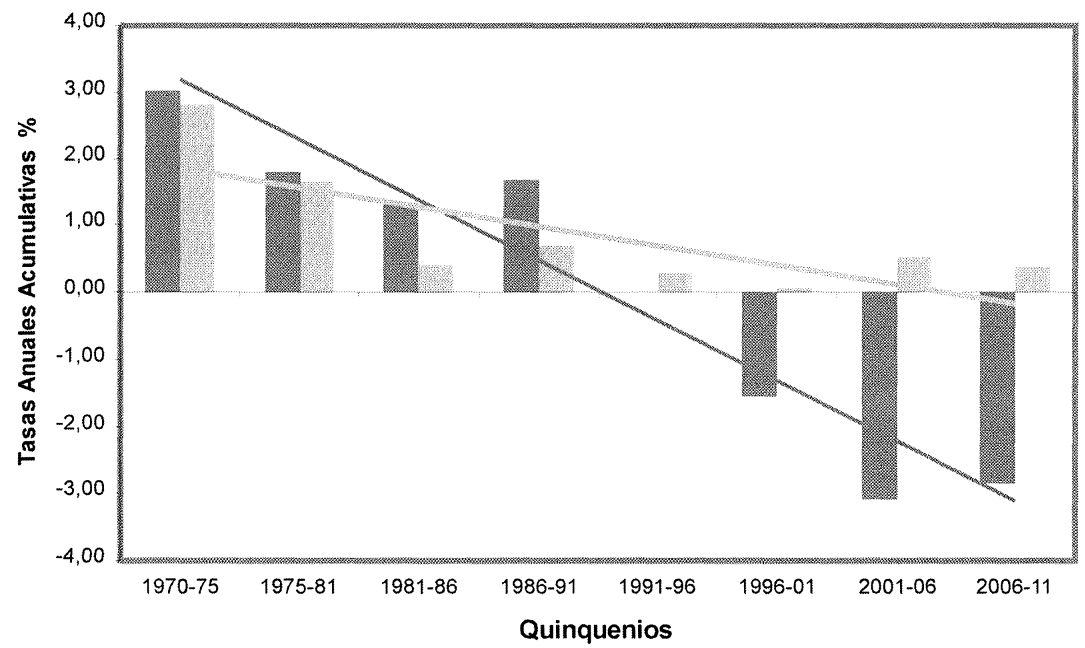

Fuente: Cuadro I.

FIGURA 1.-Comparación de las tendencias de crecimiento de la población de jóvenes (gris oscuro) y de la población total (gris claro) en la Comunidad de Madrid. 
que los jóvenes en los años 80 y 90 tuvieron que «hacerse hueco» en el insuficiente y escasamente dotado espacio que se había destinado al grupo de jóvenes en los 70. Por el contrario, en los próximos quinquenios los espacios creados supondrán una existencia mucha más holgada para las menguantes generaciones que se vayan incorporando al grupo.

\section{La estructura por edades es el principal factor de crecimiento}

Establecida la tendencia del grupo de jóvenes en la Comunidad de Madrid, interesa conocer cómo influyen en ella los distintos componentes del crecimiento. Para ello, utilizando una ecuación compensatoria,

$$
P_{15-29}^{t+1}=P_{15-29}^{t}-P_{15-29}^{t}+P_{15-29}^{t}-P_{10-24}^{t, t+1}-d_{10-24}^{t, t+1}+S M_{15-29}^{t+1}
$$

se han calculado en el Cuadro III la dimensión de las defunciones, el saldo neto migratorio y el efecto de las entradas y las salidas de individuos

\section{CuAdro III}

CÁLCULO DE LOS COMPONENTES DE CRECIMIENTO PARA EL GRUPO DE 15 A 29 AÑOS DE EDAD EN LA COMUNIDAD DE MADRID

\begin{tabular}{|c|c|c|c|c|}
\hline & $\begin{array}{c}\text { Muertos del } \\
\text { quinquenio } \\
\boldsymbol{d}_{10-24}^{t, t+1}\end{array}$ & $\begin{array}{c}\text { Saldo } \\
\text { migratorio } \\
S M_{15-29}^{t+1}\end{array}$ & $\begin{array}{c}\text { Entradas- } \\
\text { salidas } \\
P_{10-14}^{t}-P_{25-29}^{t}\end{array}$ & $\begin{array}{l}\text { Crecimiento } \\
P_{15-29}^{t+1}-P_{15-29}^{t}\end{array}$ \\
\hline $1970-75$ & 1.487 & 105.744 & 35.632 & 139.889 \\
\hline $1975-81$ & 1.751 & 38.988 & 56.337 & 93.574 \\
\hline $1981-86$ & 1.943 & -1.954 & 79.575 & 75.678 \\
\hline $1986-91$ & 2.037 & 41.475 & 61.559 & 100.997 \\
\hline $1991-96$ & 2.082 & 14.078 & -12.281 & -285 \\
\hline 1996-01 & 1.895 & 32.067 & -125.732 & -95.560 \\
\hline 2001-06 & 1.611 & 32.784 & -202.992 & -171.819 \\
\hline 2006-11 & 1.369 & 43.953 & -178.237 & -135.653 \\
\hline
\end{tabular}

Fuente: Padrón municipal de 1975. Estadística de Población de la Comunidad de Madrid, 1996. Tomo I: Características Demográficas Básicas. (A través de Anteojo.) Proyecciones de población y de hogares de la Comunidad de Madrid, 1996-2011. Tomo I: Proyecciones básicas por sexo y edad. Elaboración propia. Los fallecimientos en cada período se han estimado a partir de las tasas específicas de 1996. 
en el grupo a partir de la dinámica de las diferentes generaciones en cada uno de los quinquenios considerados.

El componente migratorio sólo es preponderante en el quinquenio 1970-75, después predomina la propia dinámica de la estructura por edad. Hasta 1996 son más numerosos los efectivos que entran en el grupo juvenil que los que superan los 29 años. A partir de 1996 la llegada de las generaciones posteriores a 1975, menguadas por el descenso de la fecundidad, hace que el saldo de entradas y salidas sea negativo y adquiera el protagonismo de los cambios. La todavía hoy decreciente natalidad anuncia la continuidad de la tendencia regresiva, incluso más allá del periodo aquí considerado.

\section{El «envejecimiento» del grupo}

La comparación de los fragmentos de pirámide correspondientes a esta franja de edad expresa también con claridad un proceso de enve-

\section{CUADRO IV}

EVOLUCIÓN DE LOS GRUPOS DE JÓVENES EN LA COMUNIDAD DE MADRID

\begin{tabular}{llll}
\hline & $\mathbf{1 5 - 1 9}$ & $\mathbf{2 0 - 2 4}$ & $\mathbf{2 5 - 2 9}$ \\
\hline 1970 & 287.581 & 297.567 & 283.742 \\
1975 & 359.150 & 320.827 & 328.802 \\
1981 & 400.757 & 365.640 & 335.956 \\
1986 & 411.952 & 396.837 & 369.242 \\
1991 & 444.966 & 425.962 & 408.100 \\
1996 & 400.260 & 451.298 & 427.185 \\
2001 & 311.024 & 415.914 & 456.245 \\
2006 & 259.884 & 325.445 & 426.035 \\
2011 & 253.505 & 278.045 & 344.161 \\
\hline
\end{tabular}

Fuente: Padrón municipal de 1975. Estadística de Población de la Comunidad de Madrid 1996. Tomo I: Características Demográficas Básicas. (A través de Anteojo.) Proyecciones de población y de hogares de la Comunidad de Madrid 1996-2011. Tomo I: Proyecciones básicas por sexo y edad.

$$
-690-
$$


jecimiento. La silueta de 1996 ya refleja los efectos del descenso de la fecundidad en la segunda mitad de los setenta. En el 2011 nos encontramos con una estructura «envejecida» que, como se indicaba anteriormente, anuncia una clara tendencia regresiva. Las generaciones de reemplazo son siempre más pequeñas que las reemplazadas (Fig. 2).

Otro aspecto que conviene considerar es la continuidad del perfil de la pirámide. Las irregularidades que se observan en 1970 son el reflejo de los movimientos migratorios, y van desapareciendo en la medida en que estos se suavizan o, como ocurre en las proyecciones, se hacen constantes.

Este «envejecimiento» es el efecto de la caída de la fecundidad y expresa el desfase temporal en la evolución de los distintos grupos quinquenales. El grupo de 15 a 19 comenzó a decrecer en 1991, mientras que el de 25 a 29 no lo hará hasta el 2001. Y ello supone que mientras que en 1991 los tres grupos tienen un tamaño similar, en el 2001 el grupo de los más jóvenes sólo representa poco más de las dos terceras partes del de los mayores, con una diferencia de 150.000 individuos.

\section{Menor presión de la demanda juvenil}

Estableciendo la relación que existe entre la dinámica de estos grupos y determinadas demandas sociales, se confirma, por ejemplo, que la oferta docente de segunda enseñanza ha comenzado a tener holguras a partir de 1991 y en el 2006 habrá que atender una demanda potencial que apenas alcanzará al 60\% de la que existía en 1991. Por su parte, la demanda demográfica universitaria, que podría identificarse con el grupo de 20 a 24, comenzó a disminuir a partir de 1996 y en tan sólo quince años habrán desaparecido cuatro de cada diez potenciales demandantes de plaza.

La intensidad de estas reducciones podría verse modificada por la elevación de los índices de escolarización, pero, tras los espectaculares aumentos alcanzados en los últimos veinte años, no cabe esperar que puedan seguir creciendo mucho más. La educación y el alargamiento de los años de estudio han pasado a ser consideradas por los jóvenes como un bien en sí mismo (Toharia, L.,1994), pero, en todo caso, dados los niveles actuales, el aumento de los índices de escolarización nunca podría llegar a compensar el efecto de la disminución del tamaño de las generaciones, ni siquiera en la demanda universitaria. En conclusión, la 
1970

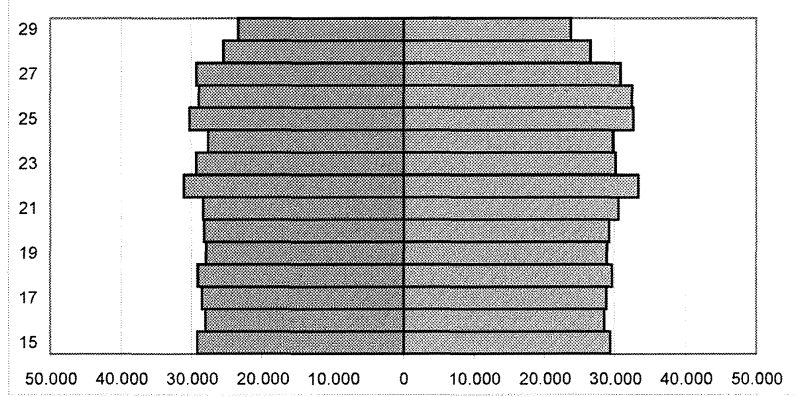

1996

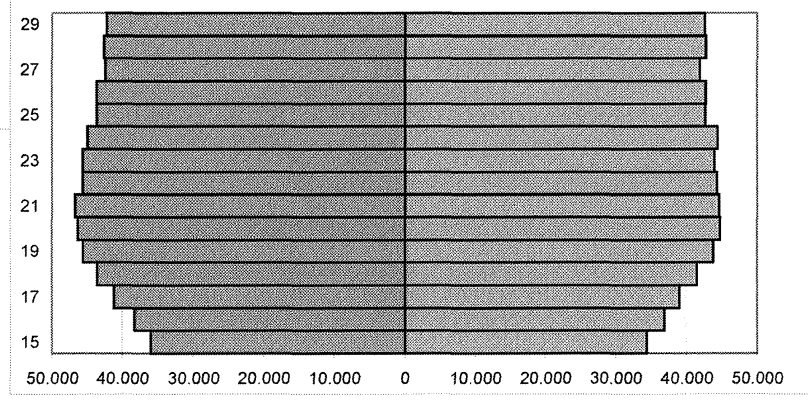

2011

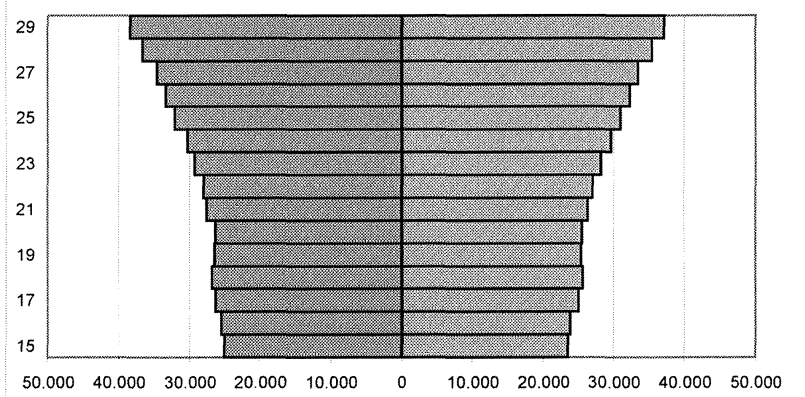

Fuente: Censo de 1970, Padrón de 1966 y Proyecciones de Población y de Hogares de la Comunidad de Madrid, 1966-2011.

Figura 2.-Evolución de la de la estructura por edad de los jóvenes de la Comunidad de Madrid.

$$
-692-
$$


dinámica demográfica del grupo de jóvenes hace que vayan quedando atrás las situaciones de masificación y posibilita mejoras cualitativas en los diferentes niveles del sistema educativo.

También a título de ejemplo, se realiza a continuación una aproximación a lo que el grupo de 20 a 29 años puede representar como generador de demanda potencial de vivienda por la formación de nuevos hogares, tras la emancipación. Utilizando el método de la Tasa de Jefatura de Hogar (Vinuesa, J., 1994), se obtienen unos flujos de generación de nuevos hogares cuyo volumen va claramente decreciendo para el grupo de 20 a 24 (Cuadro V). Además de la reducción del grupo es muy importante el descenso de la tasa de jefatura. En el grupo de 25 a 29, las oscilaciones de los flujos reflejan igualmente el descenso de la tasa y las variaciones de los efectivos del grupo. Puede concluirse que a partir del 2001, de no producirse una recuperación de las tasas de jefatura, para lo que sería necesario un adelanto de la nupcialidad o de la emancipación, se consolidará, acentuándose, la tendencia decreciente de la demanda potencial de vivienda entre los jóvenes.

La disminución de las Tasas de Jefatura de Hogar está estrechamente relacionada con la correspondiente a la nupcialidad y ésta viene a ser la principal causa del retraso de la fecundidad entre las mujeres jóvenes, al que se hará referencia más adelante.

\section{CUADRo V}

FLUJOS DE GENERACIÓN DE HOGARES POR EDAD DE LA PERSONA PRINCIPAL

\begin{tabular}{lrrr}
\hline & $\mathbf{2 0 - 2 4}$ & $\mathbf{2 5 - 2 9}$ & $\mathbf{2 0 - 2 9}$ \\
\hline $1981-1986$ & 23.477 & 67.996 & 91.473 \\
$1986-1991$ & 22.073 & 67.707 & 89.781 \\
$1991-1996$ & 14.531 & 55.251 & 69.782 \\
$1996-2001$ & 13.398 & 68.652 & 82.050 \\
$2001-2006$ & 10.483 & 64.381 & 74.865 \\
$2006-2011$ & 8.953 & 52.374 & 61.328 \\
\hline
\end{tabular}

Fuente: Cuadro 7 y Estadística de Población de la Comunidad de Madrid.1996. Tomo III: Hogares, familias y núcleos. Comunidad de Madrid. Madrid (1998). Las tasas de Jefatura de Hogar a partir de 1996 se han mantenido constantes. Elaboración propia.

$$
-693-
$$


No hay que ignorar en todo caso el decisivo papel que juega en todo esto el grado de dificultad para acceder a una primera vivienda. Como es conocido, en España el mercado de vivienda es especialmente caro y por razones culturales consolidadas a lo largo de los últimos 50 años, es un mercado sesgado hacia la propiedad. Esta circunstancia supone una especial dificultad, muy especialmente en regiones como Madrid, para la emancipación de los jóvenes, que cuando apenas han comenzado a tener ingresos propios, con una vida laboral cada vez menos estable, tienen que hacer frente a la compra de un bien con un precio diez veces mayor que sus ingresos anuales.

Un dato complementario del anterior puede ser la proporción de soltería. Como puede observarse en el Cuadro VI, es en el grupo de mujeres de 25 a 29 en el que se registran una mayor incremento de la soltería, lo cual está muy relacionado con el incremento de las tasas de actividad femeninas y con la disminución de las tasas de fecundidad. Hechos, que como se verá más adelante, vienen a remarcar el protagonismo de las mujeres en los principales cambios sociodemográficos del grupo de los jóvenes considerado como reemplazo generación.

Estos rasgos del comportamiento reciente de los jóvenes deben ser relacionados también con cambios en las pautas de consumo. Las mujeres de 25 a 29 años han pasado de una tasa de actividad de 46,8 en 1981 a otra de 79,6 en 1996. Al mismo tiempo han reducido a un tercio su fe-

CUAdRo VI

PROPORCIÓN DE SOLTEROS

\begin{tabular}{|c|c|c|c|c|c|c|}
\hline & \multicolumn{2}{|c|}{ 20-24 } & \multicolumn{2}{|c|}{ 25-29 } & \multicolumn{2}{|c|}{ Total } \\
\hline & Hombres & Mujeres & Hombres & Mujeres & Hombres & Mujeres \\
\hline 1970 & 90,3 & 71,7 & 44,2 & 29,5 & 52,8 & 49,0 \\
\hline 1981 & 81,7 & 63,7 & 34,8 & 25,9 & 50,2 & 46,6 \\
\hline 1986 & 91,6 & 79,3 & 50,7 & 36,5 & 50,3 & 46,1 \\
\hline 1991 & 94,1 & 85,1 & 62,7 & 46,4 & 49,3 & 44,6 \\
\hline 1996 & 96,8 & 92,4 & 74,5 & 59,1 & 49,9 & 44,9 \\
\hline
\end{tabular}

Fuente: Estadísticas de Población de la Comunidad de Madrid. 1996. Tomo I: Características demográficas Básicas. 
cundidad. A pesar de las cifras de desempleo, los jóvenes, en su conjunto, han escalado muchos peldaños en su capacidad de renta y han reducido considerablemente sus cargas familiares.

Destacada presencia demográfica de jóvenes, todavía

Realizadas algunas valoraciones del grupo en sí mismo, interesa ahora considerarlo en relación con el resto de la población. Medir su presencia y su participación como grupo con aptitudes y comportamientos específicos.

Según los datos del Cuadro II, para el 2011 el grupo de los jóvenes queda reducido prácticamente a las dos terceras partes de su tamaño en 1996, con un volumen similar al de 1970, cuando la población total tenía un millón y medio de habitantes menos. Dicho de otra manera, la población juvenil, tras haber llegado a representar casi un 26 por ciento en 1991 sólo supondrá el 16,6 por ciento de la población total en el 2011

Conviene señalar, sin embargo, que la presencia demográfica de los jóvenes en la Comunidad de Madrid es ligeramente mayor que en el conjunto nacional y, como puede verse en los datos del Cuadro VII, el efecto de la caída de la fecundidad le afecta en menor grado. En el

\section{CUADRO VII}

PORCENTAJES DE LOS GRUPOS DE EDAD CON RESPECTO A LA POBLACIÓN TOTAL

\begin{tabular}{lrrrrrrr}
\hline & \multicolumn{2}{c}{ Comunidad de Madrid } & & \multicolumn{3}{c}{ España } \\
\cline { 2 - 3 } & $\mathbf{1 9 9 1}$ & $\mathbf{1 9 9 6}$ & $\mathbf{2 0 0 1}$ & $\mathbf{1 9 9 1}$ & $\mathbf{1 9 9 6}$ & $\mathbf{2 0 0 1}$ \\
\hline $15-19$ & 8,99 & 7,97 & 6,12 & & 8,63 & 7,72 & 5,96 \\
$20-24$ & 8,61 & 8,99 & 8,18 & & 8,37 & 8,45 & 7,51 \\
$25-29$ & 8,25 & 8,51 & 8,98 & & 8,02 & 8,27 & 8,39 \\
$15-29$ & 25,85 & 25,46 & 23,28 & & 25,02 & 24,44 & 21,87 \\
\hline
\end{tabular}

Fuente: Censo de 1991, Padrón de 1996. Proyecciones de Población y Hogares de la Comunidad de Madrid 1996-2011 y Proyecciones de la Población de España calculada a partir del Censo de Población de 1991. Total Nacional: 1990-2020. 
2001 la proporción del grupo de jóvenes en Madrid será casi un punto y medio mayor que en la población española.

Con objeto de remarcar la importancia de este grupo en la población de la Comunidad de Madrid, es también oportuno señalar que España, por lo que a la proporción del grupo de jóvenes se refiere, ocupaba en 1996 el primer lugar entre los países europeos. La media de la Unión Europea es de $22,2 \%$ y Francia $(21,6 \%)$ y Alemania $(21,3)$ están claramente por debajo (Eurostat,1997).

\section{Capacidad de sustitución de la población activa}

Tal como se indicaba al principio, uno de los focos de interés del estudio geodemográfico del grupo de los jóvenes es medir su capacidad para «alimentar» en el futuro el grupo de edad potencialmente activa. A estos efectos se ha considerado que lo oportuno es relacionar el grupo de 20-24 años con el de 60-64. Entre los 25 y los 64 años de edad se registran las tasas de actividad más elevadas y se intenta evaluar la capacidad de sustitución de las generaciones de los jóvenes sobre este grupo. Partiendo del supuesto de que el grupo de 20-24 se incorporará al mundo laboral a lo largo del siguiente quinquenio, mientras que el de 6064 lo abandonará, los índices de sustitución de la población en edad activa superiores a 1 , reflejan crecimiento del potencial demográfico de la fuerza de trabajo. Los valores por debajo de la unidad, además de una merma de la población potencialmente activa supondrán su envejecimiento.

$$
I s^{t+1}=\frac{P_{20-24}^{t}}{P_{60-64}^{t}}
$$

donde $I s^{t+1}$ es el índice de sustitución de la población en edad activa para el final del quinqueni $t, t+1, P_{20-24}^{t}$ es la población de 20 a 24 años al comienzo del quinquenio y $P_{60-64}^{t}$ es la población de 60 a 64 años al comienzo del quinquenio.

El cálculo de estos Índices de Sustitución para el conjunto de la Comunidad, a la que se considera como ámbito del mercado de trabajo, muestran con claridad los cambios que se van a ir produciendo en los sucesivos quinquenios (Cuadro VIII). 
CUADRO VIII

ÍNDICES DE SUSTITUCIÓN DE LA POBLACIÓN EN EDAD ACTIVA

\begin{tabular}{lccc}
\hline & Hombres & Mujeres & Total \\
\hline 1986 & 2,30 & 1,87 & 2,06 \\
1991 & 2,00 & 1,65 & 1,81 \\
1996 & 1,90 & 1,58 & 1,73 \\
2001 & 1,89 & 1,60 & 1,74 \\
2006 & 1,89 & 1,59 & 1,73 \\
2011 & 1,31 & 1,11 & 1,20 \\
2016 & 1,03 & 0,85 & 0,93 \\
\hline
\end{tabular}

Fuente: Censos y Padrones. Proyecciones... Elaboración propia.

De una situación, como la de 1981-86, en la que por cada salida por jubilación se producen dos incorporaciones de jóvenes, se llega a no cubrir el reemplazo a partir del 2011. La menguada generación de los que nacieron durante la Guerra Civil, que se jubilarán a partir del 2001, hace que se retrase el efecto de la caída de la fecundidad sobre el índice de sustitución hasta el periodo 2006-2011.

Pero los efectos de este debilitamiento demográfico se ven atemperados si en lugar de considerar simplemente la población total, se toman en cuenta los datos de población activa. En este caso el índice se calcula mediante la siguiente relación

$$
I s P a^{t+1}=\frac{P_{20-24}^{t} * T a_{25-29}^{t+1}}{P a_{60-24}^{t}}
$$

donde $I s P a^{t+1}$ es el índice de sustitución de la población activa para el final del quinquenio $t, t+1, P_{20-24}^{t}$ es la población de 20 a 24 años al comienzo del quinquenio, $T a^{t+1}{ }_{25-29}$ es la tasa de actividad del grupo 25-29 al final del quinquenio y $P a_{60-64}^{t}$ es la población activa de 60 a 64 años al comienzo del quinquenio.

Como quiera que las tasas de actividad para los individuos de 25-29 son crecientes, por la progresiva incorporación de las mujeres al mer-

$$
-697-
$$


cado laboral, y mucho mayores que las tasas de actividad para el grupo de 60 a 64, los Indices de Sustitución para la población activa (IsPa) presentan unos valores que muestran una holgada, aunque también decreciente, capacidad de reemplazo. Incluso para el periodo 2011-2016 habrá 2,6 incorporaciones por cada salida de actividad (Cuadro IX).

Es especialmente significativa la intensidad del efecto sustitución por parte de la población femenina. Entre el 1996 y el 2006 puede haber, al final de cada quinquenio, mas de 8 nuevas trabajadoras de 25-29 años por cada una de 60-64 que abandone la actividad. Incluso después del 2011 la capacidad derivada de la tasa de actividad supone un índice de sustitución superior a 4 para las mujeres.

No debe pasarse por alto que en 1996 la proporción de mujeres de 25 a 29 años con titulación universitaria era del 29,18\%, frente a un 5,65\% de las mujeres de 60 a 64 . Lo cual tiene especial importancia en el proceso de sustitución de la población activa si se considera que en las sociedades más desarrolladas la economía está basada cada vez más en el conocimiento

No debe pasarse por alto tampoco que en 1996 la proporción de mujeres de 25 a 29 años con título universitario era del $29,18 \%$, frente a

CuAdro IX

ÍNDICES DE SUSTITUCIÓN DE LA POBLACIÓN EN EDAD ACTIVA

\begin{tabular}{lccc}
\hline & Hombres & Mujeres & Total \\
\hline 1986 & 3,28 & 8,72 & 3,93 \\
1991 & 3,38 & 9,03 & 4,53 \\
1996 & 3,26 & 8,04 & 4,62 \\
2001 & 3,36 & 8,64 & 4,72 \\
2006 & 3,47 & 8,66 & 4,79 \\
2011 & 2,47 & 5,80 & 3,37 \\
2016 & 1,97 & 4,28 & 2,60 \\
\hline
\end{tabular}

Fuente: Censos y Padrones. Proyecciones. Estadística de Población de la Comunidad de Madrid, 1996. Tomo I: Estudios y Actividad económica de la población. Las tasas de actividad a partir del 2001 se han proyectado de forma lineal a partir del comportamiento de los años anteriores. 
un 5,63\% para las mujeres de 60-64 años. Lo cual tiene especial importancia si se considera que en las sociedades más desarrolladas la economía está basada, cada vez más, en el conocimiento.

\section{Decreciente participación de las mujeres jóvenes en la fecundidad}

Como es bien sabido las tasas de fecundidad por grupos de edad han evolucionado en España desde 1976 dando lugar al retraso del calendario y, por tanto, a la consiguiente disminución de la participación de las jóvenes en la fecundidad. Si a ello se suma el efecto de la evolución regresiva de la composición por edades, el fenómeno se agudiza.

Como puede apreciarse (Cuadro X), los porcentajes de nacimientos de madres de 20-24 se han reducido drásticamente en los últimos veinticinco años. Ello es debido fundamentalmente a la disminución de la Tasa Especifica de Fecundidad que pasa de 142,35, en 1975, a 16,68, en 1996. Si se mantienen constantes las tasas de fecundidad de 1996, se ob-

CuAdro X

PARTICIPACIÓN DE LAS MUJERES JÓVENES EN LA FECUNDIDAD DE LA COMUNIDAD DE MADRID

\begin{tabular}{|c|c|c|c|c|c|c|}
\hline & \multicolumn{3}{|c|}{ Mujeres de 20 a 24 años } & \multicolumn{3}{|c|}{ Mujeres de 25 a 29 años } \\
\hline & $\underset{\text { Naci- }}{\text { mientos }}$ & $\begin{array}{c}\% \text { de } \\
\text { nacimientos } \\
\text { sobre el total }\end{array}$ & $\begin{array}{c}\text { \% de } \\
\text { mujeres } \\
\text { sobre el total } \\
\text { en edad fértil }\end{array}$ & $\underset{\text { Naci- }}{\text { mientos }}$ & $\begin{array}{c}\% \text { de } \\
\text { nacimientos } \\
\text { sobre el total }\end{array}$ & $\begin{array}{c}\% \text { de } \\
\text { mujeres } \\
\text { sobre el total } \\
\text { en edad fértil }\end{array}$ \\
\hline 1975 & 23.065 & 25,12 & 15,18 & 34.053 & 37,06 & 15,74 \\
\hline 1981 & 17.149 & 25,02 & 15,79 & 24.194 & 35,29 & 14,69 \\
\hline 1986 & 11.793 & 20,58 & 16,06 & 22.129 & 38,62 & 15,35 \\
\hline 1991 & 6.758 & 13,88 & 15,73 & 18.832 & 38,69 & 15,31 \\
\hline 1996 & 3.702 & 7,90 & 15,97 & 14.760 & 31,49 & 15,31 \\
\hline 2001 & 3.407 & 7,08 & 14,89 & 15.596 & 32,39 & 16,39 \\
\hline 2006 & 2.666 & 5,61 & 12,01 & 14.534 & 30,56 & 15,74 \\
\hline 2011 & 2.280 & 5,24 & 10,70 & 11.734 & 26,95 & 13,24 \\
\hline
\end{tabular}

Fuente: Censos y Padrones de Población. Movimientos Naturales de la Población de la Comunidad de Madrid. Delgado (1990). Proyecciones... Elaboración propia. 
tiene, para el 2011, un número de nacimientos que no llega a ser la décima parte de los que producían este grupo en 1976. La participación de las madres de estas edades en la fecundidad madrileña se ha visto reducida a menos de un tercio en los últimos veinte años y sólo uno de cada veinte niños nacidos en el 2011 tendrá una madre de 20 a 24 años.

El grupo de 25 a 29 años refleja una disminución de la fecundidad similar a la del conjunto. No se manifiesta en sus datos el retraso de la maternidad de las más jóvenes que se ve contrarrestado por el propio aplazamiento de la maternidad entre las mujeres de este grupo, que, a su vez, hace que las máximas tasas se localicen entre las mujeres de 30 a 34 años. La Tasa Específica de Fecundidad de este grupo ha pasado de 202,65 en 1975 a 69,39 en 1996, pero esta es una disminución sólo ligeramente superior a la del conjunto. La proporción de nacimientos de mujeres de esta edad sólo se hace menor a partir de 1991 y se verá afectada por la estructura por edad a partir del 2006.

En resumen puede destacarse que prácticamente desaparece la fecundidad de las mujeres de 20 a 24 años que hasta 1981 ocupaban el segundo lugar por orden de importancia, con una cuarta parte de los nacimientos. Los grupos de mujeres jóvenes han cedido el protagonismo al de las mujeres de 30 a 34 años que de ocupar el tercer lugar han pasado al primero de forma destacada.

Si se simplifica la comparación el resultado es aún más elocuente: en 1981 un $60,31 \%$ de los niños de Madrid nacían de madres de veinte

\section{CUADro XI}

JERARQUIZACIÓN DE LOS GRUPOS DE EDAD POR SU PARTICIPACIÓN EN LA FECUNDIDAD

\begin{tabular}{cccc}
\hline & $\mathbf{1 9 8 1}$ & $\mathbf{1 9 9 6}$ & $\mathbf{2 0 1 1}$ \\
\hline $\mathbf{1 .}^{\circ}$ & $25-29(35,29)$ & $30-34(41,61)$ & $30-34(45,40)$ \\
$\mathbf{2 .}^{\circ}$ & $20-24(25,02)$ & $25-29(31,49)$ & $25-29(26,95)$ \\
$\mathbf{3 .}^{\circ}$ & $30-34(22,87)$ & $35-39(15,12)$ & $35-39(18,50)$ \\
$\mathbf{4 .}^{\mathbf{0}}$ & $35-39(9,54)$ & $20-24(7,90)$ & $20-24(5,24)$ \\
\hline
\end{tabular}

Fuente: Censos y Padrones de Población. Movimientos Naturales de la Población de la Comunidad de Madrid. Delgado (1990). Proyecciones... Elaboración propia. 
años y un 32,41\% de madres de treinta. En el 2011, de mantenerse las actuales pautas de fecundidad, la proporción se habrá invertido y los porcentajes serán 32,19 y 63,90 respectivamente. (Fig. 3)

\section{La dinámica de crecimiento está marcada} por el desarrollo metropolitano

$\mathrm{Al}$ repetir este análisis para ámbitos más pequeños los resultados que se obtienen reflejan especialmente la importancia del proceso de metropolitanización en la dinámica demográfica. Sólo con distinguir el municipio de Madrid, la Corona Metropolitana y el Resto de los municipios no metropolitanos, se observa que, junto a factores comunes como el descenso de la fecundidad, la inercia de la estructura por edades, modelada principalmente por los desplazamientos migratorios propios de la expansión urbana hacia la periferia metropolitana, marca diferencias espaciales en la cronología de la dinámica del grupo de jóvenes (Fig. 4).

En el centro metropolitano, la población joven del municipio de Madrid comenzó a disminuir a partir de 1991, y quedará reducida en el 2011 a poco más de la mitad. En la Corona Metropolitana la inflexión no se producirá hasta el 2001 y en el Resto será a partir del 2006.

El estudio del desglose de estos crecimientos en sus distintos componentes viene a apoyar la teoría que aquí se defiende y que considera que las pautas de los desarrollos urbanos locales, dentro del marco del modelo de expansión metropolitana vivido en la Comunidad de Madrid, son el principal factor modelador de la dinámica demográfica.

1981

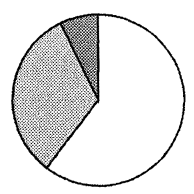

1996

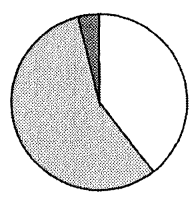

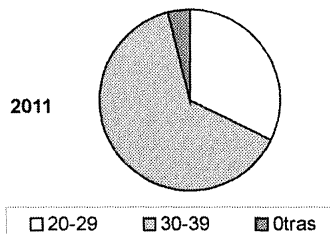

Fuente: Censo y Padrones. Margarita Delgado, 1990, Movimientos Naturales de la Población. Comunidad de Madrid.

Figura 3.-Evolución de la participación de las madres de 20 a 29 años en la fecundidad de la Comunidad de Madrid. 


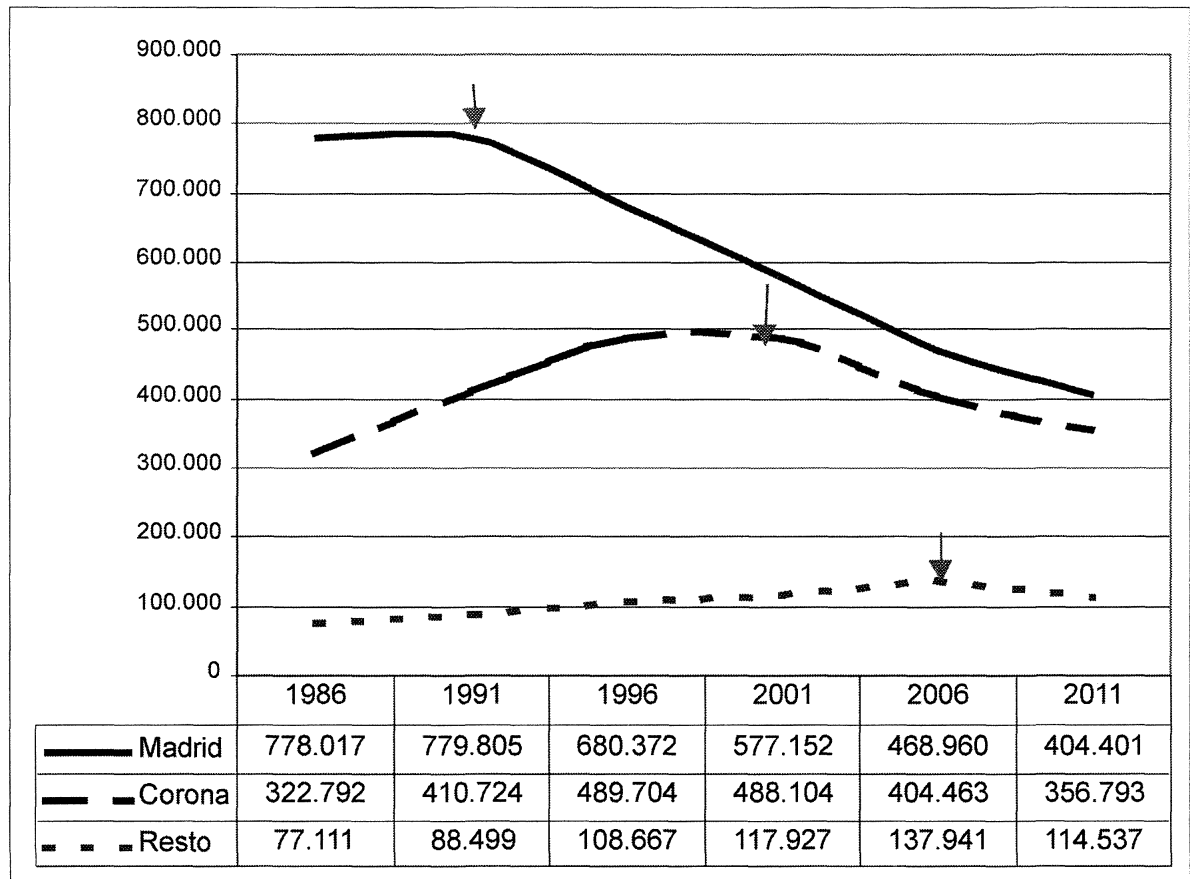

Fuente: Padrones, Censos y Proyecciones...

FIGURA 4.-Evolución del grupo j15 a 29 por grandes zonas.

En todos los casos las tendencias regresivas comienzan con los reemplazos con saldos fuertemente negativos, que se producen por la dinámica de la propia estructura, al ser mucho menores las generaciones que se incorporan al grupo de jóvenes que las que le abandonan. La merma de las entradas es el efecto diferido del descenso de la fecundidad, mientras que los calendarios de las migraciones debidas a la expansión metropolitana inciden especialmente sobre el tamaño del grupo de 25 a 29 años.

Desconcentración espacial de los jóvenes

Las diferentes pautas de crecimiento hacen que se modifiquen también de forma notable los pesos relativos de los grupos de jóvenes de 
CUADro XII

CÁLCULO DE LOS COMPONENTES DE CRECIMIENTO DEL GRUPO DE 15 A 29 AÑOS EN DIFERENTES ÁMBITOS DE LA COMUNIDAD DE MADRID

\begin{tabular}{|c|c|c|c|c|}
\hline & \multicolumn{4}{|c|}{ Municipio de Madrid } \\
\hline & $\begin{array}{c}\text { Muertos } \\
\text { quinquenio }\end{array}$ & $\begin{array}{c}\text { Saldo } \\
\text { migratorio }\end{array}$ & $\begin{array}{c}\text { Entradas- } \\
\text { salidas }\end{array}$ & Crecimiento \\
\hline $1986-91$ & 1.297 & -7.955 & 11.040 & 1.788 \\
\hline $1991-96$ & 1.167 & -28.345 & -69.921 & -99.433 \\
\hline $1996-01$ & 941 & 5.522 & -107.801 & -103.220 \\
\hline $2001-06$ & 754 & 10.804 & -118.242 & -108.192 \\
\hline \multirow[t]{3}{*}{$2006-11$} & 632 & 20.443 & -84.370 & -64.559 \\
\hline & \multicolumn{4}{|c|}{ Corona Metropolitana } \\
\hline & $\begin{array}{c}\text { Muertos } \\
\text { quinquenio }\end{array}$ & $\begin{array}{c}\text { Saldo } \\
\text { migratorio }\end{array}$ & $\begin{array}{c}\text { Entradas- } \\
\text { salidas }\end{array}$ & Crecimiento \\
\hline $1986-91$ & 609 & 40.789 & 47.752 & 47.752 \\
\hline $1991-96$ & 769 & 22.407 & 57.342 & 57.342 \\
\hline 1996-01 & 789 & 8.684 & -9.495 & -9.495 \\
\hline $2001-06$ & 669 & -1.709 & -81.263 & -81.263 \\
\hline \multirow[t]{3}{*}{$2006-11$} & 545 & 25.516 & -72.641 & -72.641 \\
\hline & \multicolumn{4}{|c|}{ Resto no Metropolitano } \\
\hline & $\begin{array}{c}\text { Muertos } \\
\text { quinquenio }\end{array}$ & $\begin{array}{c}\text { Saldo } \\
\text { migratorio }\end{array}$ & $\begin{array}{c}\text { Entradas- } \\
\text { salidas }\end{array}$ & Crecimiento \\
\hline $1986-91$ & 131 & 8.762 & 2.757 & 11.388 \\
\hline $1991-96$ & 146 & 20.016 & 298 & 20.168 \\
\hline $1996-01$ & 165 & 17.861 & -8.436 & 9.260 \\
\hline $2001-06$ & 169 & 35.496 & -15.313 & 20.014 \\
\hline $2006-11$ & 189 & 11 & -23.226 & -23.404 \\
\hline
\end{tabular}

Fuente: Padrón municipal de 1975. Estadística de Población de la Comunidad de Madrid 1996. Tomo I: Características Demográfícas Básicas. (A través de Anteojo.) Proyecciones de población y de hogares de la Comunidad de Madrid 1996-2011. Tomo I: Proyecciones básicas por sexo y edad. Elaboración propia. Los fallecimientos en cada periodo se han estimado a partir de las tasas específicas de 1996 
acuerdo con la dinámica demográfica y la expansión metropolitana (Fig. 5). En 1986 puede apreciarse una ligera concentración de jóvenes en la Capital, con un 66\% frente al 64\% de la población total. En 1996 la concentración se traslada a la Corona que pasa a tener el $38 \%$ de los jóvenes de la Comunidad y el 34\% de la población. Por último, en el 2006 el coeficiente de localización más alto se produce en el Resto de los municipios no metropolitanos. En los 20 años que consideramos, la Capital pasó del $66 \%$ al $46 \%$ de los jóvenes de la Comunidad. Trece de los puntos perdidos por la Capital los gana la Corona y los otros 7, el Resto. Así pues, al igual que está ocurriendo con el conjunto de la población, la de jóvenes tiende a desconcentrarse pero a mayor ritmo.

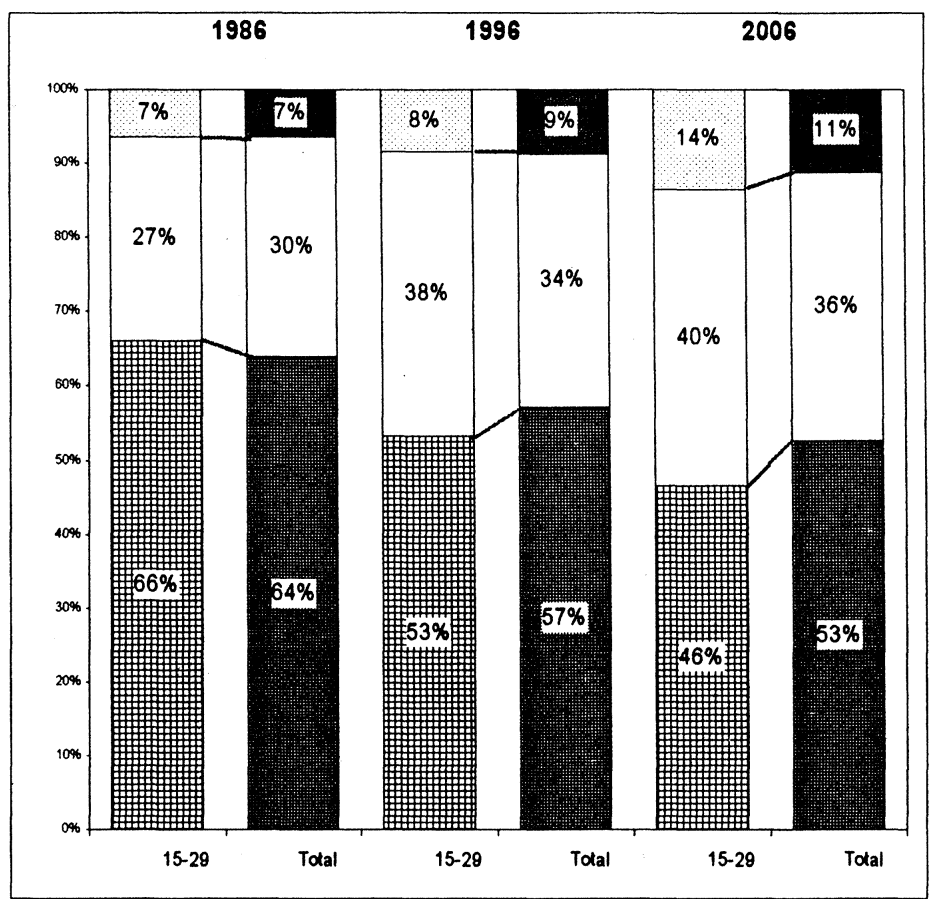

Fuente: Padrones y Proyecciones...

Figura 5.-Distribución de los jóvenes y de la población total en los distintos ámbitos (Madrid, Corona y Resto). 
Este proceso de desconcentración es consecuencia de la distribución espacial del mercado inmobiliario que, siendo el principal factor explicativo de la redistribución espacial de la población de la Comunidad de Madrid (VINUESA, J. 1999), dirige los flujos migratorios, especialmente de jóvenes, hacia zonas con oferta de viviendas más asequibles por más alejadas del centro (Comunidad de Madrid, 1991). Los datos del Cuadro XIII muestran cómo disminuyen los índices de soltería con el alejamiento del centro metropolitano. La mayor proporción de solteros en el municipio de la capital es el claro reflejo de las dificultades que el mercado inmobiliario opone a la emancipación de los jóvenes.

\section{Gran diversidad de situaciones y fuertes oscilaciones} en la dinámica de las poblaciones de jóvenes

Al hacer un análisis más detallado, considerando las poblaciones de ámbitos más reducidos y los grupos quinquenales de edad, se observan una gran diversidad de situaciones, que básicamente son consecuencia de los desfases temporales de unos procesos de dinámica demográfica muy similares.

Comenzando con el porcentaje del grupo de jóvenes (Fig. 6), resulta que en la «Almendra» formada por los distritos centrales de Madrid los jóvenes sólo representan el 21,2\%. En los distritos periféricos la proporción está en torno al $25 \%$ y en la Corona Metropolitana es más alta y

CuAdro XIII

PROPORCIÓN DE SOLTEROS

\begin{tabular}{lcccccc}
\hline & \multicolumn{2}{c}{ Mun. de Madrid } & \multicolumn{2}{c}{ Corona Metrop. } & \multicolumn{2}{c}{ Resto no Metrop. } \\
Edad & Hombres & Mujeres & Hombres & Mujeres & Hombres & Mujeres \\
\hline 25 & $92,6 \%$ & $84,7 \%$ & $84,6 \%$ & $68,0 \%$ & $80,4 \%$ & $59,6 \%$ \\
26 & $88,6 \%$ & $80,0 \%$ & $75,3 \%$ & $55,5 \%$ & $70,5 \%$ & $46,5 \%$ \\
27 & $83,4 \%$ & $70,2 \%$ & $63,7 \%$ & $44,3 \%$ & $61,4 \%$ & $39,8 \%$ \\
28 & $76,1 \%$ & $61,6 \%$ & $53,4 \%$ & $35,2 \%$ & $50,7 \%$ & $32,9 \%$ \\
29 & $69,6 \%$ & $54,4 \%$ & $43,1 \%$ & $27,6 \%$ & $43,7 \%$ & $26,2 \%$ \\
\hline
\end{tabular}




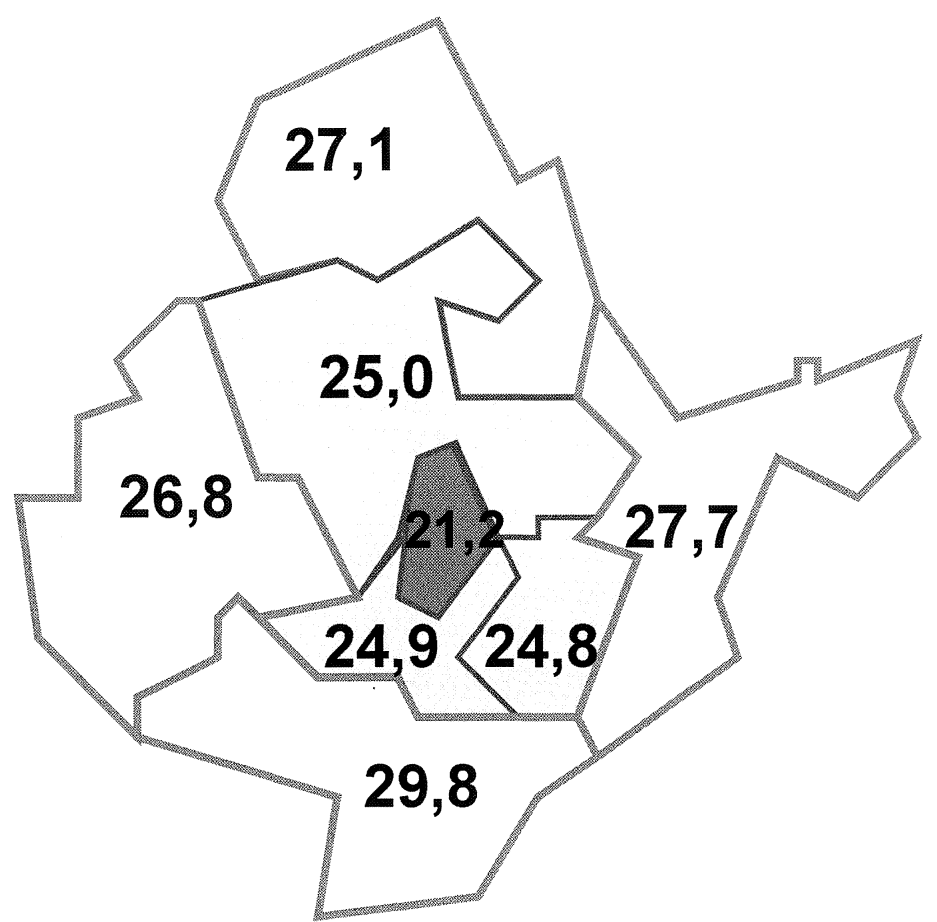

Fuente: Cuadro XII.

Figura 6.-Porcentajes de jóvenes por grandez zonas del Área Metropolitana, 1996.

diversa, destacando el 29,8 de la Corona Sur. Casi nueve puntos porcentuales separan los valores de esta zona de los del centro de la capital.

El índice de Reposición General $\left(P_{10-14}^{1996}-P_{25-29}^{1996}\right) / P_{15-29}^{1996} * 100$, expresa en tantos por ciento el crecimiento, entre 1996 y el 2001, de la población de jóvenes debido sólo a la propia estructura por edad. Hay que tener en cuenta que a lo largo del quinquenio salen del grupo los que en 1996 tenían 25-29 y se incorpora los que tenían 10-14. Los primeros corresponden a la generación de los nacidos entre 1965 y 1971, periodo de alta natalidad, mientras que los segundos pertenecen a la de los nacidos entre 1981 y 1986, con valores de fecundidad muy reducidos. 
Todos los grupos quinquenales considerados en este análisis corresponden a generaciones nacidas entre 1965 y 1986, periodo en el que progresivamente, sobre todo a partir de 1976, la fecundidad ha ido disminuyendo. Sólo los saldos migratorios que hayan podido actuar específicamente sobre los diferentes grupos en los distintos ámbitos, al compás del desarrollo metropolitano, han podido alterar el comportamiento debido a la fecundidad.

Los resultados muestran valores negativos para todas las zonas (Fig. 7). Si bien hay grandes diferencias entre la intensidad de las pérdidas en el municipio de Madrid y las que se registran en la Corona $\mathrm{Me}-$ tropolitana. La diversidad mencionada permite que incluso haya algunos municipios como Tres Cantos, Rivas-Vaciamadrid o Coslada, con Indices de Reposición claramente positivos.

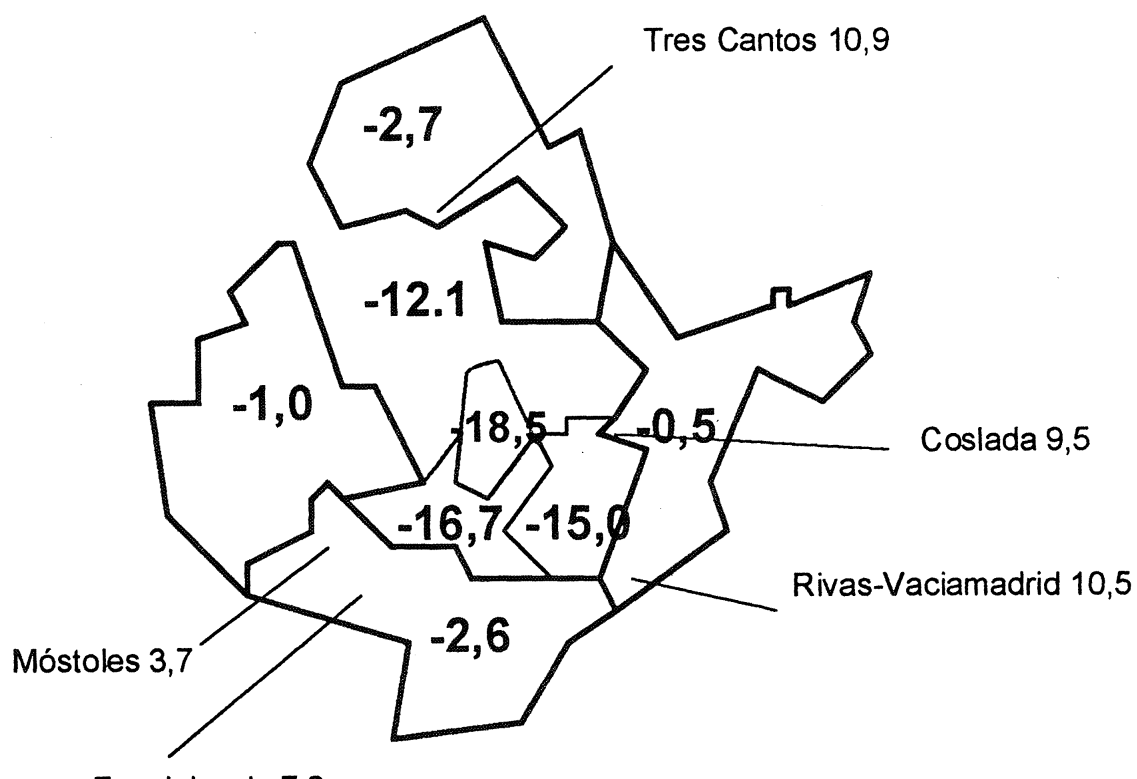

Fuenlabrada 7,8

Fuente: Cuadro XII.

Figura 7.-Índices de Reposición 15-29 (1996-2001). 
Los Índices de Reposición de los diferentes grupos de edad muestran que tienden a disminuir con carácter general e intensamente los efectivos más jóvenes, mientras que aumentarán en la mayor parte de las zonas los efectivos del grupo de mayor edad.

Los valores del Índice de Reposición del grupo de 15 a 19 años $\left(P_{10-14}^{1996}-P_{15-29}^{1996}\right) / P_{15-19}^{1996} * 100$ reflejan en general reducciones entre un quinta y una cuarta parte (Fig. 8), si bien algunos municipios como Alcorcón, Leganes o Getafe perderán 4 de cada 10 individuos de este grupo, lo que se deja notar sobre todo en la reducción de la demanda de plazas escolares. Sin embargo tambien hay municipios en los que la estructura hace que este grupo aumente su tamaño. Tales son los casos de Rivas, San Fernado y Tres Cantos que manifiestan así desarrollos urbanos más recientes.

La distribución del Índice de Reposición del grupo intermedio, 20-24, $\left(P_{15-14}^{1996}-P_{20-24}^{1996}\right) / P_{20-24}^{1996} * 100$ es el que muestra una mayor diversidad (Fig. 9). Sólo en el muncipio de la capital se producen pérdidas im-

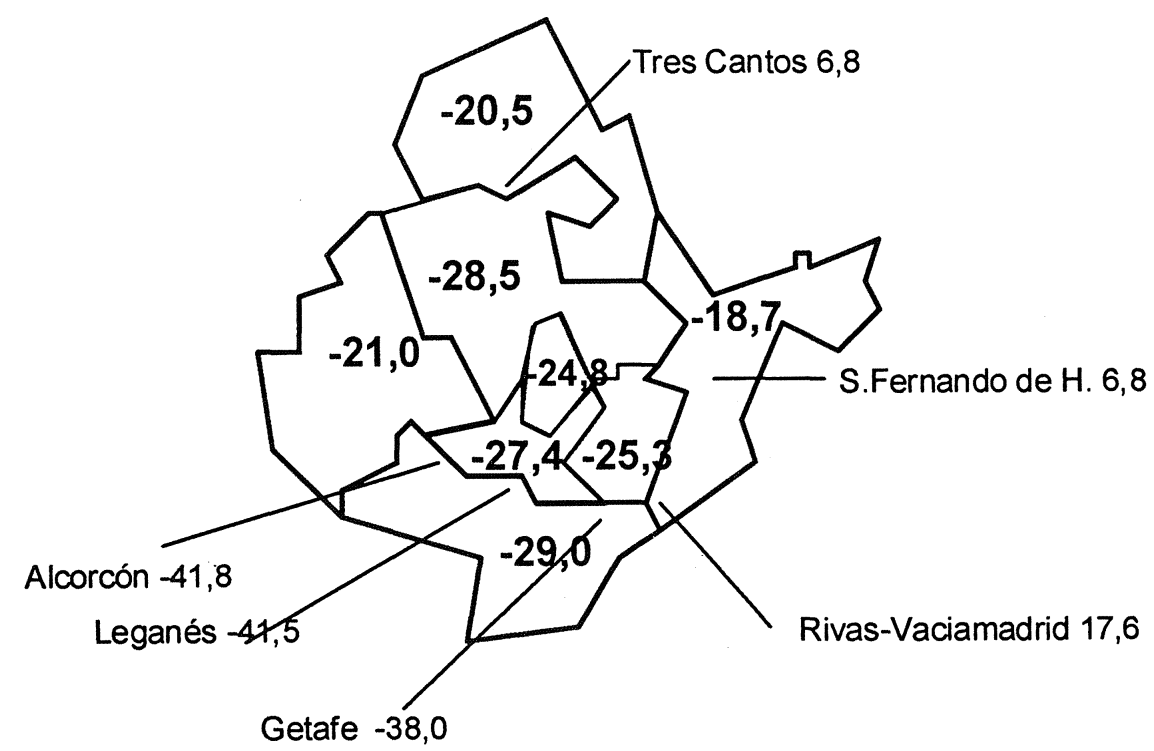

Fuente: Cuadro XII.

Figura 8.-Índices de Reposición 15-19 (1996-2001). 


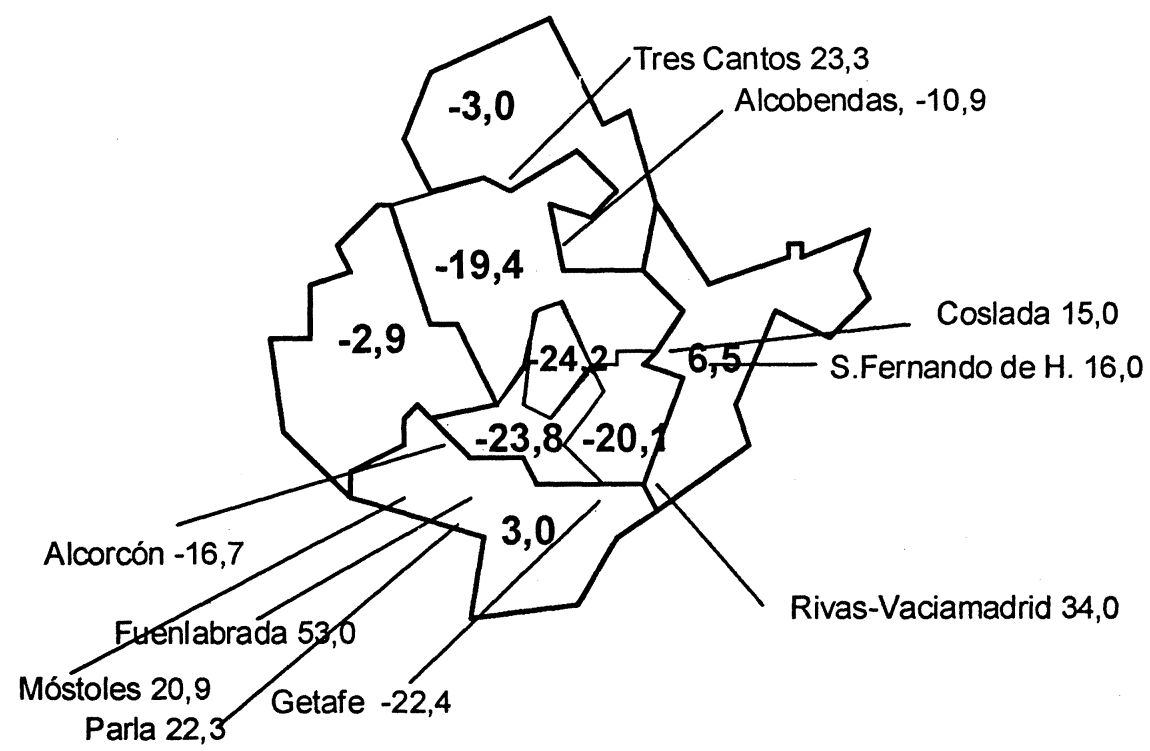

Fuente: Cuadro XII.

Figura 9.-Índices de Reposición 20-24 (1996-2001).

portantes. En la Corona decrece levemente la mitad noroccidental y aumenta, tambien levemente, las zonas Sur y Este. Los valores de los municipios muestran sin embargo bastantes casos de cambios muy bruscos. Además de Fuenlabrada, cuya estructura hace que este grupo aumente más de un $10 \%$ al año, otros varios van a crecer más de un $20 \%$ en el quinquenio.

Para el grupo de $25-29\left(P_{20-24}^{1996}-P_{25-29}^{1996}\right) / P_{25-29}^{1996} * 100$, resulta un panoráma claramente positivo. Sólo disminuye la Almendra Central y levemente las Periferias Sur y Este. En toda la Corona Metropoltana la inercia de la estructura va a hacer que crezca considerablemente el grupo de jóvenes en el que estadísticamente se hacen efectivas la nupcialidad, la emancipación, la demanda de la primera vivienda o el nacimiento del primer hijo. Nuevamente aparecen municipios con comportamientos muy difirentes. Desde los que registrarán aumentos superiores al 50\%, como Coslada, hasta los que van a perder uno de cada 3 individuos, como San Fernando. 


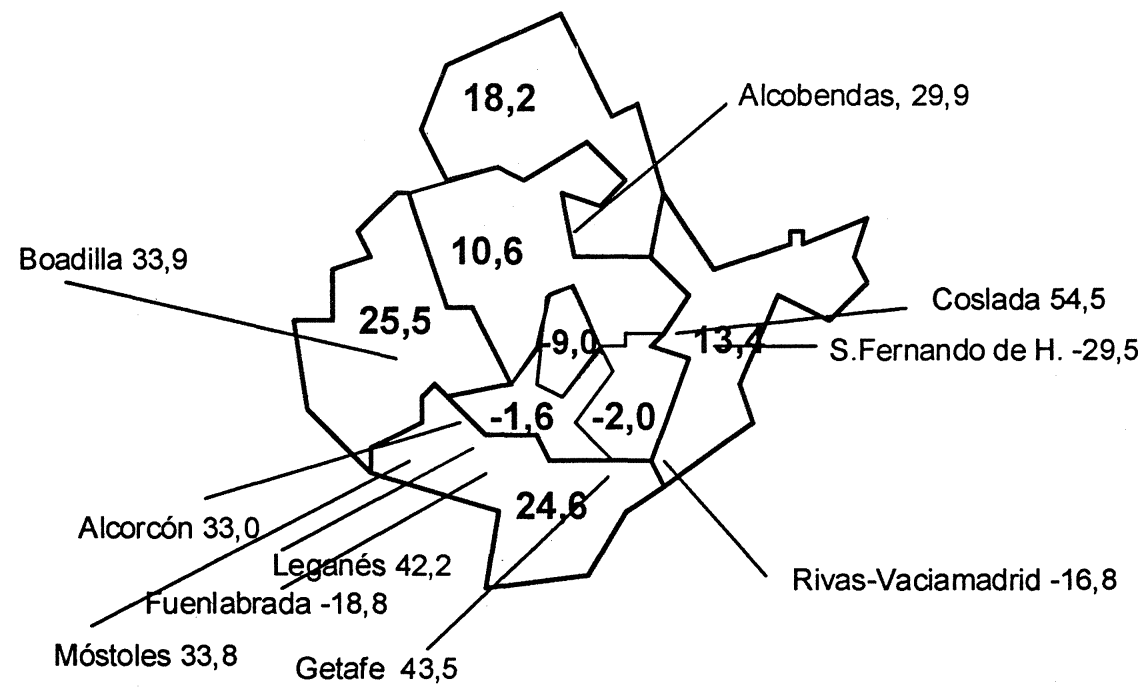

Fuente: Cuadro XII.

Figura 10.-Indices de Reposición 25-29 (1996-2001).

Estos índices de reposición y, sobre todo los indicadores a nivel municipal (Cuadro XIV), hacen evidentes algunos de los efectos más perversos del modelo de desarrollo metropolitano de Madrid, que da lugar a una dinámica demográfica caracterizada por los fuertes fluctuaciones en el tamaño de grupos concretos de población como los que aquí se estudian. Este comportamiento demográfico es necesariamente causante de situaciones urbanísticas conflictivas al producirse rápidos crecimientos de las necesidades de vivienda o de la demada de primer empleo $\mathrm{y}$, especialmente en los ámbitos locales, fuertes fluctuaciones de las demanda de equipamientos y servicios urbanos.

Estos valores, correspondientes al quinquenio que finalizará en el 2001, expresan la extrema variabilidad de unas poblaciones especificas dentro de un contexto de estacionaridad. Efectivamente, mientras el conjunto de la población de la Comunidad de Madrid registra unos leves crecimientos, consecuencia de unas tasas de natalidad y mortalidad estables y muy similares, y de un saldo neto migratorio también irrelevante, en los grupos de edad aquí analizados y en ámbitos territoriales concretos

$$
-710-
$$


CUADro XIV

INDICADORES DE LA POBLACIÓN JOVEN

POR GRUPOS QUINQUENALES, ZONAS Y MUNICIPIOS (1996-2001)

\begin{tabular}{|c|c|c|c|c|c|}
\hline & \multirow{2}{*}{$\begin{array}{l}\text { Porcentaje } \\
\text { de jóvenes }\end{array}$} & \multicolumn{4}{|c|}{ Índices de reposición } \\
\hline & & $15-19$ & 20-24 & $25-29$ & $15-29$ \\
\hline TOTAL & 25,5 & $-24,7$ & $-11,3$ & $-05,6$ & $-09,8$ \\
\hline Municipio de Madrid & 23,7 & $-26,8$ & $-22,3$ & $-1,0$ & $-15,8$ \\
\hline Almendra Central & 21,2 & $-24,8$ & $-24,2$ & $-9,0$ & $-18,5$ \\
\hline Periferia Noroeste & 25,0 & $-28,5$ & $-19,4$ & 10,6 & $-12,1$ \\
\hline Periferia Este & 24,8 & $-25,3$ & $-20,1$ & $-2,0$ & $-15,0$ \\
\hline Periferia Sur & 24,9 & $-27,4$ & $-23,8$ & $-1,6$ & $-16,7$ \\
\hline Corona Metropolitana & 28,6 & $-24,9$ & 2,4 & 21,3 & $-1,9$ \\
\hline Corona Metropolitana Norte & 27,1 & $-20,5$ & $-3,0$ & 18,2 & $-2,7$ \\
\hline Alcobendas & 29,8 & $-30,4$ & $-10,9$ & 29,9 & $-5,6$ \\
\hline Colmenar Viejo & 24,4 & $-8,9$ & $-4,1$ & 2,3 & $-3,5$ \\
\hline San Sebastián de los Reyes & 27,6 & $-21,2$ & 2,3 & 13,8 & $-2,5$ \\
\hline Tres Cantos & 20,6 & 6,8 & 23,3 & 3,4 & 10,9 \\
\hline Corona Metropolitana Este & 27,7 & $-18,7$ & 6,5 & 13,4 & $-0,5$ \\
\hline Alcalá de Henares & 29,1 & $-23,8$ & $-2,9$ & 17,6 & $-3,9$ \\
\hline Coslada & 26,5 & $-20,6$ & 15,0 & 54,5 & 9,5 \\
\hline Mejorada del Campo & 26,6 & $-7,2$ & 44,8 & $-7,1$ & 7,6 \\
\hline Paracuellos de Jarama & 26,7 & $-10,0$ & $-11,3$ & 20,4 & $-1,2$ \\
\hline Rivas-Vaciamadrid & 19,9 & 17,6 & 34,0 & $-16,8$ & 10,5 \\
\hline San Fernando de Henares & 25,1 & 6,8 & 16,0 & $-29,5$ & $-5,0$ \\
\hline Torrejón de Ardoz & 29,3 & $-24,4$ & 7,8 & 9,1 & $-3,4$ \\
\hline Velilla de San Antonio & 24,9 & 1,2 & 4,7 & $-36,2$ & $-14,0$ \\
\hline Corona Metropolitana Sur & 29,8 & $-29,0$ & 3,0 & 24,6 & $-2,6$ \\
\hline Alcorcón & 31,8 & $-41,8$ & $-16,7$ & 33,0 & $-10,3$ \\
\hline Getafe & 31,0 & $-38,0$ & $-22,4$ & 43,5 & $-8,7$ \\
\hline Fuenlabrada & 26,6 & $-0,3$ & 53,0 & $-18,8$ & 7,8 \\
\hline Leganés & 30,8 & $-41,5$ & $-10,3$ & 42,2 & $-6,9$ \\
\hline Móstoles & 29,3 & $-29,2$ & 20,9 & 33,8 & 3,7 \\
\hline Parla & 30,8 & $-26,7$ & 22,3 & 14,5 & 0,8 \\
\hline Pinto & 27,5 & $-21,6$ & 8,2 & $-2,5$ & $-5,7$ \\
\hline Corona Metropolitana Oeste & 26,8 & $-21,0$ & $-2,9$ & 25,5 & $-1,0$ \\
\hline Boadilla del Monte & 28,8 & $-26,4$ & $-7,3$ & 33,9 & $-2,4$ \\
\hline Brunete & 24,3 & $-8,3$ & $-11,8$ & $-14,7$ & $-11,9$ \\
\hline
\end{tabular}


CuAdro XIV (Continuación)

\begin{tabular}{|c|c|c|c|c|c|}
\hline & \multirow{2}{*}{$\begin{array}{l}\text { Porcentaje } \\
\text { de jóvenes }\end{array}$} & \multicolumn{4}{|c|}{ Índices de reposición } \\
\hline & & $15-19$ & 20-24 & 25-29 & $15-29$ \\
\hline Majadahonda & 27,7 & $-22,3$ & 4,1 & 29,5 & 1,3 \\
\hline Pozuelo de Alarcón & 27,0 & $-25,3$ & $-11,0$ & 21,9 & $-5,7$ \\
\hline Rozas de Madrid (Las) & 24,9 & $-12,1$ & 2,9 & 25,0 & 3,7 \\
\hline Villanueva de la Cañada & 25,5 & $-5,8$ & 2,5 & 3,8 & 0,1 \\
\hline Villanueva del Pardillo & 23,4 & $-12,7$ & 28,9 & $-21,2$ & $-4,0$ \\
\hline Villaviciosa de Odón & 28,6 & $-28,8$ & $-3,0$ & 54,9 & 1,7 \\
\hline Área Metropolitana & 25,6 & $-25,9$ & $-12,1$ & 7,1 & $-10,0$ \\
\hline M. no metropolitanos & 24,4 & $-12,0$ & $-2,1$ & $-9,1$ & $-7,8$ \\
\hline Aranjuez & 25,3 & $-18,6$ & $-3,6$ & 7,7 & $-5,0$ \\
\hline Arganda del Rey & 27,4 & $-18,7$ & $-2,6$ & 12,8 & $-3,3$ \\
\hline Collado Villalba & 25,1 & $-12,4$ & 3,4 & $-16,3$ & $-9,0$ \\
\hline Valdemoro & 28,4 & $-12,6$ & 7,9 & $-14,7$ & $-7,0$ \\
\hline Promedio & 27,0 & $-19,0$ & 1,0 & 10,0 & $-4,0$ \\
\hline Desviación Estándar & 2,7 & 12,4 & 17,4 & 21,2 & 7,1 \\
\hline
\end{tabular}

Fuente: Estadística de la población de la Comunidad de Madrid. 1996. Características demográficas básicas. A través de Anteojo.

son frecuentes las inflexiones muy bruscas. Esta situación no es específica del quinquenio valorado, sino que, por el contrario, perdura con el modelo de desarrollo metropolitano, produciendo unos comportamientos casi erráticos que se convierten en un importante obstáculo para la planificación urbana, para una eficiente asignación de los recursos y, a la postre, merman la calidad de la vida urbana en un espacio que difícilmente podrá estar adecuadamente dotado en consonancia con una composición por edades caracterizada por sus permanentes cambios.

\section{Conclusiones}

Una vez más se confirma que en aras de una mayor aplicabilidad es necesario estudiar la población con la adecuada desagregación espacial y agrupada en razón de sus papeles en la estructura socioeconómica. 
Los grupos de población de jóvenes además de ser considerados por su propia presencia, tienen un destacado significado como protagonistas de relevos generacionales, especialmente en relación con la actividad económica y con la fecundidad. Con carácter general, las generaciones que se incorporan al grupo de jóvenes van siendo siempre menores que las que lo abandonan.

La población joven de la Región metropolitana de Madrid tiene un comportamiento similar al del conjunto de España y presenta una tendencia claramente regresiva, si bien es algo mayor su presencia todavía. Los jóvenes de los próximos años se encontrarán con unas ofertas de todo tipo que les permitirán unas existencias mucho más holgadas y menos competitivas que las que han tenido sus predecesores.

La dinámica de los grupos de jóvenes es consecuencia, sobre todo, de la estructura por edades, modelada por el rápido descenso de la fecundidad y, en mucha mayor medida, por los flujos migratorios intraprovinciales propios del proceso de expansión metropolitana.

La evolución del grupo está modificando sustancialmente las necesidades y demandas de los jóvenes, que también protagonizan importantes cambios de comportamiento. El sistema educativo es, sin duda, el más aliviado. La merma en la oferta de nuevos trabajadores por la evolución demográfica, se ve en buena parte compensada por el incremento de las tasas de actividad de las mujeres de 25 a 29 años, siendo muy importante que los jóvenes que se incorporan al mundo laboral tienen un nivel de formación muy superior al de los que lo abandonan.

La demanda de primera vivienda se ve aminorada, tanto por la reducción de las generaciones de jóvenes emancipables, como por el retraso de la nupcialidad. La carestía del mercado de la vivienda y el modelo de tenencia en propiedad son un factor clave en estos cambios y en la reducción de la fecundidad.

El retraso de la fecundidad, que en realidad es disminución, sintetiza los principales cambios de las jóvenes en la sociedad del último cuarto de siglo. Las mujeres de 20 a 24 años prácticamente han dejado de participar en la fecundidad y las de 25 a 29, que tradicionalmente ha asumido el mayor protagonismo, pasa a segundo lugar al reducir a un tercio su tasa de fecundidad. Más que nunca hay que buscar la trabazón entre fecundidad y vida laboral. Asimilado el principio de que la educación es un bien en sí mismo; alcanzada la igualdad entre hombres y mujeres a través del sistema educativo; el paso siguiente no puede ser 
otro que la equiparación en la participación en la actividad. Pero los cambios que se están produciendo no son sólo la consecuencia de la búsqueda de la irrenunciable igualdad de las mujeres; de su plena capacidad para decidir. El modelo social y cultural exige progresivamente unos mayores niveles de ingresos y se hacen necesaria la aportación económica de la mujer en el hogar. Sin negar el tradicional efecto negativo del crecimiento de la actividad femenina sobre la fecundidad, el aumento de las tasas de empleo entre las mujeres puede convertirse en el factor necesario para que aumente la fecundidad.

El predominio del hogar tradicional choca con el cambio de papel de la mujer y de ello se resiente la fecundidad. La mujer deberá recibir mucho más apoyo, dentro y fuera del hogar para que aumente la fecundidad.

El estudio al nivel local, pone de manifiesto la importancia de la inercia demográfica, los movimientos migratorios del proceso de urbanización en los decenios pasados y la redistribución espacial en consonancia con el modelo de expansión metropolitana producen una enorme variedad de comportamientos, con la constante de fuertes cambios de tamaño de grupos específicos de población, lo que inevitablemente se traduce en conflictividad urbanística y merma en la calidad del espacio urbano.

\section{BIBLIOGRAFÍA}

CoMunidad DE MADRID (1991): La juventud de la Comunidad de Madrid. Conocer para Actuar. Atlas Sociodemográfico, Madrid, Comunidad de Madrid

- (1998): «Proyecciones de Población y de hogares de la Comunidad de Madrid. 1996-2011». Tomo I: Proyecciones Básicas por sexo y edad. Madrid, Instituto de Estadística, Comunidad de Madrid.

DELGADO, M. (1990): La fecundidad española por grupos de edad, 1975-1985, Instituto de Demografía, CSIC, Madrid.

EUROSTAT (1997): Demographics Statistics, Bruselas, Luxemburgo.

FERnÁNDEZ CoRdón, J. A. (1993): «La población y la fecundidad de la Comunidad de Madrid», Informe Monográfico del Tomo I de los Censos de Población y Vivienda de 1991, Comunidad de Madrid, Madrid.

FLAQUER, Lluis (1997): «La emancipación familiar de los jóvenes. Revista de Estudios de Juventud, n. ${ }^{\circ} 39$, mayo de 1997.

INE (1995): Proyecciones de Población de España calculada a partir del Censo de Población de 1991. Total Nacional 1990-2020, Madrid, INE.

Martín, M., y Velarde, O. (1997): Juventud en cifras, 1996, Madrid, Ministerio de Trabajo y Asuntos Sociales, Instituto de la Juventud. 
TOHARIA, L (1994): «Estudios y Actividad Económica de la Población de la Comunidad de Madrid», Informe Monográfico del Tomo I de los Censos de Población y Vivienda de 1991, Comunidad de Madrid, Madrid.

Vienuesa, J. (edit.) (1994): Demografía. Análisis y Proyecciones, Madrid, Editorial Síntesis, Madrid.

- (1999): «La población de Madrid, transformaciones y tendencias», en Economía de las Comunidades Autónomas, Madrid, Fundación de Las Cajas de Ahorros Confederadas Para la Investigación Económica y Social, pp. 87-101.

ZÁrRAga, J. L. (1984): «La juventud en la década de los 80», en Políticas de Juventud y Administraciones Públicas, Madrid, Ministerio de Cultura, Dirección General de la Juventud, pp. 33-51.

RESUMEN: Los jóvenes en la estructura demográfica de la región metropolitana de Madrid. La dinámica de los grupos de edad entre 15 y 29 años en distintos ámbitos geográficos ayuda a explicar los efectos de las migraciones centrípetas y, posteriormente, centrífugas, que han ido conformando la región metropolitana de Madrid. La relación de la composición y del comportamiento demográfico de esos grupos con diferentes aspectos de la estructura territorial, ponen de manifiesto importantes faltas de armonía que causan conflictividad social y deficiencias en las dotaciones, rebajando la calidad del espacio urbano.

ABSTRACT: Young people within the demographic structure of Madrid metropolitan area. The age group dynamism between 15 and 29 years old located in different geographical areas helps to explain the centripetal and, later on, centrifugal migratory effects, which have been shaping Madrid metropolitan area. The relationship between those groups constitution and their demographical behaviour, having different territorial structure aspects, shows important harmony lacks, which cause social conflict and standards deficiency. Both elements lower the urban space quality.

RÉSUMÉ: Les jeunes dans la structure démographique de la région métropolitaine de Madrid. La dynamique des groupes d'âges entre 15 et 29 ans dans diverses zones géographiques permet d'expliquer les effets des migrations centripètes et, postérieurement, centrifuges qui ont constitué la région métropolitaine de Madrid. La relation entre la composition et le comportement démographique de ces groupes, et divers aspects de la structure territoriale, mettent en évidence d'importantes absences d'harmonie qui causent une conflictivité sociale et des déficiences dans les dotations, qui diminuent la qualité de vie. 